Physics and Chemistry of Liquids. 1972. Vol. 3, pp. 225-230

Copyright (c) 1972 Gordon and Breach Science Publishers

Printed in Great Britain

\title{
The Effect of a Chemical Reaction on the Damping of the Hydrodynamic Modes and the Spectrum of Scattered Light
}

\author{
H. N. W. LEKKERKERKER and W. G. LAIDLAW
}

Department of Chemistry

University of Calgary

Calgary, Alberta, Canada

and

Faculté des Sciences

Université Libre de Bruxelles

1050 Brussels, Belgium

\begin{abstract}
The roots of the hydrodynamic matrix for a two species chemically reactive system are obtained under assumptions for which the pressure fluctuations are decoupled from those in entropy and concentration. All roots are affected by the chemical reaction and attention is directed to the implications of the resulting dependence of the roots for the determination of chemical reaction rates from light scattering spectra.
\end{abstract}

The recent literature contains a number of treatments for the calculation of the spectral distribution of light scattered from a chemically relaxing fluid (see, for example, the work of Berne et al., ${ }^{(1,2)}$ Blum and Salsburg, ${ }^{(3,4,5)}$ Schurr ${ }^{(6)}$ and Knirk and Salsburg $\left.{ }^{(7)}\right)$. Although differing in level of sophistication, these treatments suggest that a chemical reaction can be taken as affecting only one of the non-propagating modes. The contribution of this mode to the light scattering spectrum has been referred to as the " chemical reaction" peak. ${ }^{(7)}$ In this letter we wish to point out that, on accounting for the concomitant presence of transport processes and the chemical reaction, the latter process affects more than one of the non-propagating modes. This coupling could be of significance in the use of light scattering spectroscopy for the determination of chemical reaction rates.

In the macroscopic calculation of the light scattering spectrum one starts from the coupled hydrodynamic equations in the set of 
variables which describe the system under investigation (see, e.g., Mountain $\left.{ }^{(8)}\right)$. We consider a fluid containing two chemical species which transform into one another via a chemical reaction, for example, a monomer-dimer equilibrium system. ${ }^{(9)}$ The pertinent equations for this system can be obtained using the methods of irreversible thermodynamics (see, e.g., Meixner ${ }^{(10)}$ and De Groot and Mazur(11) and in linearized form can be written as

$$
\begin{gathered}
\frac{\partial \rho}{\partial t}+\rho \operatorname{div} \mathbf{v}=0 \\
\rho \frac{\partial \operatorname{div} \mathbf{v}}{\partial t}=-\Delta p+\left(\frac{4}{3} \eta+\eta_{v}\right) \Delta \operatorname{div} \mathbf{v} \\
\frac{\partial \xi}{\partial t}=D\left[\Delta \xi+\frac{k_{T}}{T} \Delta T+\frac{k_{p}}{p} \Delta p\right]-\frac{1}{\tau_{T, p}}\left[\delta \xi-\left(\frac{\partial \xi}{\partial T}\right)_{p, A} \delta T-\left(\frac{\partial \xi}{\partial p}\right)_{T, A} \delta p\right] \\
\frac{\partial s}{\partial t}=\frac{\lambda}{\rho T} \Delta T+\frac{D k_{T}\left(\partial\left[\mu_{1}-\mu_{2}\right] / \partial \xi\right)_{p, T}}{T}\left[\Delta \xi+\frac{k_{p}}{p} \Delta p\right] \\
+\left(\frac{\partial s}{\partial \xi}\right)_{p, T} D\left[\Delta \xi+\frac{k_{p}}{p} \Delta p+\frac{k_{T}}{T} \Delta T\right]
\end{gathered}
$$

where, as far as possible, the notation of De Groot and Mazur ${ }^{(11)}$ has been used, with the progress variable $\xi$ taken as the mass fraction of one of the reacting species.

Further

$$
k_{p} / p=\left(\frac{\partial\left[\mu_{1}-\mu_{2}\right]}{\partial p}\right)_{T, \xi} /\left(\frac{\partial\left[\mu_{1}-\mu_{2}\right]}{\partial \xi}\right)_{T, p}
$$

and the symbol $\delta$ in front of a thermodynamic variable indicates the deviation of that variable from its equilibrium value.

We now choose a convenient set of independent variables and write Eqs. (1) to (4) in terms of them. The resulting equations are FourierLaplace transformed and the dispersion equation in the wave vector $\mathbf{k}$ and the Laplace variable $z$ obtained. Restricting ourselves to situations where

and

$$
\frac{\lambda k^{2}}{\rho c_{p, \xi}}<k c(\infty), \frac{\left(\frac{4}{3} \eta+\eta_{v}\right) k^{2}}{\rho}<k c(\infty), D k^{2}<k c(\infty)
$$

$$
\frac{1}{\tau_{T, p}}<k c(\infty)
$$


the four roots of the dispersion equation can be found, correct to first order in the coupling between the pressure fluctuations and those in entropy and concentration.

The real roots are

$$
z_{1,2}=\frac{1}{2}\left\{\left(d_{1}+d_{2}\right) \pm\left[\left(d_{1}-d_{2}\right)^{2}+4 d_{12}^{2}\right]^{1 / 2}\right\}
$$

where

$$
\begin{gathered}
d_{1}=\frac{\lambda k^{2}}{\rho c_{p, \xi}}+\frac{c_{p, i}}{c_{p, \xi} \tau_{T, p}} \\
d_{2}=D k^{2}+\frac{1}{\tau_{T, p}} \\
d_{12}=\left[D k_{T} k^{2}-\frac{(\partial \xi / \partial T)_{p, A} T}{\tau_{T, p}}\right]\left[\left(\frac{\partial\left[\mu_{1}-\mu_{2}\right]}{\partial \xi}\right)_{T, p} / T c_{p, \xi}\right]^{1 / 2}
\end{gathered}
$$

The complex roots are

$$
z_{3,4}=\left[\Gamma k^{2}+\frac{1}{2} \frac{c(\infty)^{2}-c(0)^{2}}{c(0)^{2}} \frac{1}{\tau_{s, p}}\right] \pm i k c(\infty)
$$

where $\Gamma$ has the same form as the damping factor of the Brillouin lines for a two-component non reactive system.

Condition (5) can, of course, always be met for sufficiently small $k$. For liquid mixtures the magnitude of the transport coefficients $\left(\lambda \simeq 5.10^{-3} \mathrm{cal} / \mathrm{cm} \mathrm{sec} \mathrm{deg}, \eta \simeq 10^{-2} \mathrm{~g} / \mathrm{cm} \mathrm{sec}\right.$ and $\left.D \simeq 10^{-5} \mathrm{~cm}^{2} / \mathrm{sec}\right)$ and the speed of sound $\left(c(\infty) \simeq 10^{5} \mathrm{~cm} / \mathrm{sec}\right)$ are such that, even for the maximum scattering vectors occurring in light scattering experiments $\left(k^{\max } \simeq 2.10^{5} \mathrm{~cm}^{-1}\right)$, the conditions given in Eq. (5) are generally satisfied. To meet condition (6), $k$ must be larger than some minimum value, which of course depends on $\tau_{T, p}$. In order that this minimum lies in the region of scattering vectors realized in light scattering experiments (i.e. lies below $2.10^{+5} \mathrm{~cm}^{-1}$ ), the inverse chemical relaxation time should be $10^{9} \mathrm{sec}^{-1}$ or smaller.

From Eqs. (7) to (10) it is clear that both the real roots are influenced by the chemical reaction. This may significantly affect the appearance of the central peak, but to discuss fully the effect of this coupling would require calculations of the form of the non-propagating modes and a knowledge of the pertinent derivatives of the optical dielectric constant. In this letter we give some consideration to the dependence of the real roots on $k$ and make some qualitative statements about the ensuing normal modes. 


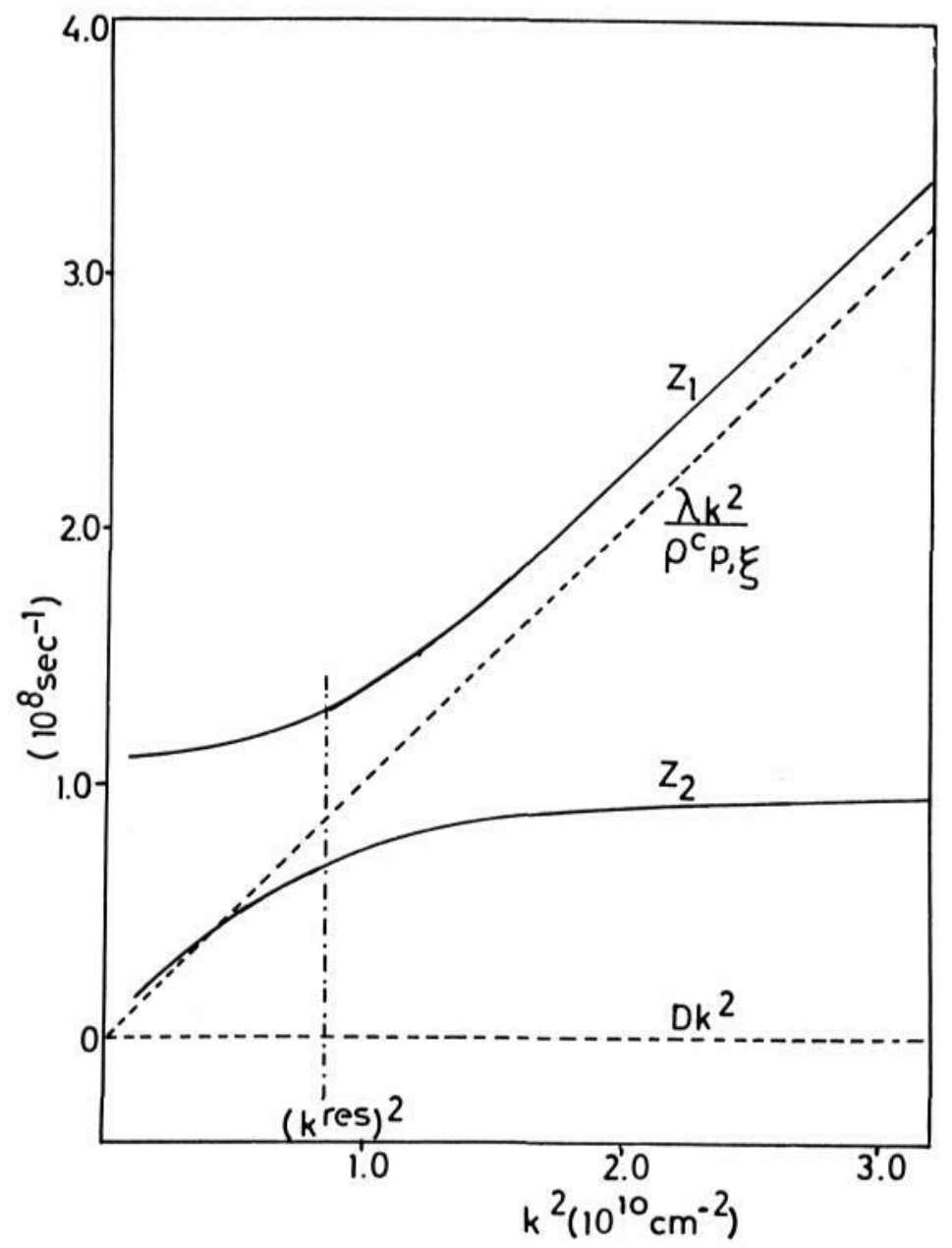

Figure 1. Dependence of the real roots on $k^{2}$.

Material Parameters: $\frac{\lambda}{\rho c_{p, \xi}}=10^{-2} \mathrm{~cm}^{2} / \mathrm{sec} ; \quad D=10^{-5} \mathrm{~cm}^{2} / \mathrm{sec}$;

$$
\begin{gathered}
\tau_{T, p}=10^{-8} \mathrm{sec} ; \quad \frac{c_{p, i}}{c_{p \cdot \xi}}=0.15 ; T=300^{\circ} \mathrm{K} ; \\
\left(\frac{\partial \xi}{\partial T}\right)_{p, A}=10^{-3} \mathrm{deg}^{-1} ; \quad\left(\frac{\partial\left[\mu_{1}-\mu_{2}\right]}{\partial \xi}\right)_{p, T} / T c_{p, \xi}=1
\end{gathered}
$$

In the present case the chemical term in $d_{12}$ is much larger than the transport term and the contribution of the latter has been neglected. 
In the figure the $k$ dependence of the real roots is depicted for a set of typical values of the material parameters. From the figure it can be seen that neither of the real roots exhibits the linear $k^{2}$ dependence typical of damping due to transport processes. Thus neither real root for the reactive system is simply displaced by a function of the inverse relaxation time from the roots for the non-reactive case. The deviation from the linear $k^{2}$ dependence is most pronounced for $k$ values for which $d_{1} \simeq d_{2}$. In analogy with physical problems with a similar mathematical structure we will refer to the $k$ value for which $d_{1}=d_{2}$ as the resonance scattering vector $\left(k^{\text {res }}\right)$.

It can readily be shown, that for $k<k^{\text {res }}$ the normal mode corresponding to the smaller real root is essentially the same as the heat conductivity mode of the two component non-reactive system with a small admixture of the mass diffusion mode, whereas the normal mode corresponding to the larger real root is essentially the same as the mass diffusion mode of the two component non-reactive system with a small admixture of the heat conductivity mode. As $k$ increases the mixing becomes more pronounced and for $k=k^{\text {res }}$ is complete. When $k \gg k^{\text {res }}$ the normal mode corresponding to the smaller root has become essentially the same as the mass diffusion mode of the two component non-reactive system with a small admixture of the heat conductivity mode whereas the normal mode corresponding to the larger real root has become principally the same as the heat conductivity mode of the two component non-reactive system with a small admixture of the mass diffusion mode.

The $k$ dependence of the real roots, especially in the region of resonance, and the concomitant mixing of the heat conduction and mass diffusion modes suggests that considerable care should be exercised in extracting the chemical relaxation time from the central component of the light scattering spectrum.

Although taking into account the presence of both transport processes and chemical reaction led to expressions for the real roots which were not linear in $k^{2}$, the real part of the complex roots is still linear in $k^{2}$. Actually the only effect of the chemical reaction is an additional term, $\left[\left(c(\infty)^{2}-c(0)^{2}\right) / c(0)^{2}\right]\left[1 / 2 \tau_{s p}\right]$ indicating that the width of the Brillouin peaks could, in principle, be used to determine the chemical relaxation time. 


\section{Acknowledgements}

The authors are grateful for the hospitality extended to them by Professor Prigogine's group. The support of the National Research Council of Canada and the sabbatical leave granted to WGL by the University of Calgary is acknowledged. The award of a Killam predoctoral fellowship and the award of a NATO Science fellowship by the Netherlands Organisation for the Advancement of Pure Research are acknowledged by HNWL.

\section{REFERENCES}

1. Berne, B. J. and Frisch, H. L., J. Chem. Phys. 47, 3675 (1967).

2. Berne, B. J., Deutch, J. M., Hynes, J. T. and Frisch, H. L., J. Chem. Phys. 49, 2864 (1968).

3. Blum, L. and Salsburg, Z. W., J. Chem. Phys. 48, 2292 (1968).

4. Blum, L. and Salsburg, Z. W., J. Chem. Phys. 50, 1654 (1969).

5. Blum, L., J. Chem. Phys. 51, 5024 (1969).

6. Schurr, J. M., J. Phys. Chem. 73, 2820 (1969).

7. Knirk, D. L. and Salsburg, Z. W., J.Chem. Phys. 54, 1251 (1971).

8. Mountain, R. D., Rev. Mod. Phys. 38, 205 (1966).

9. Freedman, E., J. Chem. Phys. 21, 1784 (1953).

10. Meixner, J., Z. Naturf. 7A, 553 (1952).

11. de Groot, S. R. and Mazur, P., Non-Equilibrium Thermodynamics, NorthHolland Publ. Co., Amsterdam, 1962. 